\title{
Universal assay for measuring vertebrate telomeres by real-time quantitative PCR
}

Stephanie F. Hudon ${ }^{1 *}$, Esteban Palencia Hurtado², James D. Beck ${ }^{3}$, Steven J. Burden ${ }^{1}$, Devin P. Bendixsen ${ }^{1}$, Kathleen R. Callery ${ }^{2}$, Jennifer S Forbey ${ }^{2}$, Lisette P. Waits ${ }^{4}$, Robert A. Miller ${ }^{5}$, Ólafur K. Nielsen ${ }^{6}$, Julie A. Heath ${ }^{2}$ and Eric J. Hayden ${ }^{2}$

${ }^{1}$ Biomolecular Sciences, Boise State University, Boise, ID 83725, USA

2 Department of Biological Sciences, Boise State University, Boise, ID 83725, USA

${ }^{3}$ Computational Science and Engineering, Boise State University, Boise, ID 83725, USA

${ }^{4}$ Department of Fish and Wildlife Sciences, University of Idaho, Moscow, ID 83844, USA

${ }^{5}$ Intermountain Bird Observatory, Boise, ID 83725, USA

${ }^{6}$ Department of Ecology, Icelandic Institute of Natural History, Garðabær 210, Iceland

* To whom correspondence should be addressed. Tel: +1 208995 3765; Email: stephaniehudon@boisestate.edu Correspondence may also be addressed to Tel: +1 650400 8439; Email: erichayden@boisestate.edu

\section{ABSTRACT}

Telomere length dynamics are an established biomarker of health and aging in animals. The study of telomeres in numerous species has been facilitated by methods to measure telomere length by real-time quantitative PCR (qPCR). In this method, telomere length is determined by quantifying the amount of telomeric DNA repeats in a sample and normalizing this to the total amount of genomic DNA. This normalization requires the development of genomic reference primers suitable for qPCR, which remains challenging in non-model organism with genomes that have not been sequenced. Here we report reference primers that can be used in qPCR to measure telomere lengths in any vertebrate species. We designed primer pairs to amplify genetic elements that are highly conserved between evolutionarily distant taxa and tested them in species that span the vertebrate tree of life. We report five primer pairs that meet the specificity and reproducibility standards of qPCR. In addition, we demonstrate how to choose the best primers for a given species by testing the primers on multiple individuals within a species and applying an established computational tool. These reference primers can facilitate the application of qPCR-based telomere length measurements in any vertebrate species of ecological or economic interest.

\section{INTRODUCTION}

The measurement of telomere lengths is an important approach used to study the health and aging of organisms. Telomeres are structures at the end of Eukaryotic chromosomes that are comprised of proteins bound to repetitive DNA sequences. Telomeres protect the ends of linear chromosomes and provide several important cellular functions (1). The length of telomere DNA shortens at each cell division, and telomere shortening can eventually lead to cellular senescence, which affects tissue function, organismal health and lifespan (2-4). Telomere lengths are considered an indicator of phenotypic quality $(5,6)$ and telomere length dynamics have been shown to have predictive power of the future success of organisms. For example, telomere shortening has been shown to predict both lifespan and reproductive success $(7,8)$, which are proxies for organismal fitness. Short telomere 
lengths have even been shown to precede extinction events (9). In addition, telomere shortening has been shown to be accelerated by various forms of stress (10-12) and telomere lengths have been used to evaluate environmental quality (13-15). The ability to monitor the well-being of organisms and to predict their future success has important applications in both wild and captive environments. In addition, understanding telomere dynamics across the vertebrate tree of life could also lead to a better understanding of the mechanisms and evolution of aging in general.

Despite the motivation for measuring telomere lengths, research remains limited to relatively few species, with the majority of studies done in avian species (16-18). Methodological challenges are one reason for the limited use of telomere length measurements. A widely used method is to measure relative telomere length by real-time quantitative PCR (qPCR). This method requires only common lab equipment and techniques, can be done in relatively high-throughput and is robust despite low sample quantity and quality $(19,20)$. Measurement of telomere length by qPCR requires primers that amplify telomere repeats, where the concentration of telomeric DNA in a sample, determined by $\mathrm{QPCR}$, is proportional to telomere length. The method also requires reference primers that amplify a non-telomeric region of the genome of interest to normalize for the total amount of genomic DNA in the sample (21). The repeating DNA sequence of telomeres (TTAGGG) is identical in all vertebrates, so the established telomere-specific primer pairs should work in any vertebrate species (22). In contrast, the genome specific reference primers optimized in one species may not work in other species because of unknown genetic differences, often requiring the design and optimization of new primers for each new species that is to be studied $(23,24)$. Reference primer development is especially challenging in non-model organisms with genomes that have not been sequenced. Despite the simplicity of measuring telomere lengths by qPCR, reference primer design may limit the adoption of this assay in newly analyzed species.

Here we report PCR primers that can be used as genomic reference primers for qPCR basedtelomere length measurements in any vertebrate species. To design a PCR assay that would work universally in vertebrates, we designed primers to amplify ultra-conserved elements (UCEs) which are genetic elements that are highly conserved between evolutionarily distant taxa (25-27). We identified primer pairs that amplify five different UCEs and also meet qPCR requirements for specificity and efficiency (28) (Figure 1A). We designed these primers to match the annealing temperature of the established telomere primers so that they can be used in the same PCR plate if desired. We found that all five primers efficiently and reproducibly amplify genomic DNA in every vertebrate sample that we tested. In addition, the targeted UCEs do not vary in copy number in the species we investigated. As a proof of concept, we used the primers to verify a significant decrease in telomere length with age in American kestrels, a species for which telomere lengths had not previously been measured. The reported qPCR primers enable the expansion of telomere length studies to new vertebrate systems for basic and applied research questions. 


\section{MATERIAL AND METHODS}

\section{Genomic DNA extraction}

DNA was extracted from blood or tissue samples using the Zymo Quick-DNA Microprep Plus Kit (\#D4074) according to the manufacture's protocols for the sample type. Blood samples from Northern goshawk and American kestrel were stored in Queen's lysis buffer $(0.01 \mathrm{M}$ Tris, $0.01 \mathrm{M} \mathrm{NaCl}, 0.01 \mathrm{M}$ EDTA, and $1 \%$ n-lauroylsarcosine, $\mathrm{pH}$ 7.5) prior to extraction. Human DNA was from a buccal swab. DNA from a red-eared slider was extracted from a shell fragment. DNA from sea squirt was extracted from whole organisms. All other DNA samples were extracted from muscle tissue. All animals were treated in accordance with Boise State University animal care and use policies where applicable. DNA purity was assessed by $260 / 280 \mathrm{~nm}$ absorbance ratio (NanoDrop). DNA was quantified by absorbance at $260 \mathrm{~nm}$ and the concentration was normalized to $2 \mathrm{ng} / \mu \mathrm{l}$ in $10 \mathrm{mM}$ Tris- $\mathrm{HCl} \mathrm{pH} 8.5$ and $0.1 \mathrm{mM}$ EDTA.

\section{Quantitative PCR}

Quantitative PCR (qPCR) was performed using a Roche LC96. A standard curve for each primer pair was included in triplicate on each qPCR plate. Genomic DNA from the organism of interest which had the highest concentration, measured by UV absorbance, was used for the standard curve. The standard curve was prepared by seven serial dilutions (1:5). Reactions were carried out in $20 \mu \mathrm{L}$ volumes containing approximately $8 \mathrm{ng}$ DNA for unknown samples (or $4 \mu \mathrm{l}$ of varying concentrations of DNA for serial dilutions), $10 \mu$ of 2x Biotium Fast Plus EvaGreen® qPCR Master Mix, 10 pmol each of forward and reverse primers ( $500 \mathrm{nM}$ final primer concentration) and water up to $20 \mu \mathrm{l}$. For UCE and telomere primers, the two-step thermal cycling profile was $95^{\circ} \mathrm{C}$ for $2 \mathrm{~min}$, followed by 40 cycles of $95^{\circ} \mathrm{C}$ for $5 \mathrm{~s}$ and $55^{\circ} \mathrm{C}$ for $30 \mathrm{~s}$, with signal acquisition at the end of the $55^{\circ} \mathrm{C}$ step and melt curves generated by increasing temperatures from 72 to $95^{\circ} \mathrm{C}$, in $0.5^{\circ} \mathrm{C}$ steps, with a $30 \mathrm{~s}$ dwell period per step at the end of the thermal cycling. Reactions with previously reported primers for 36B4 in mice contained a $250 \mathrm{nM}$ final concentration of forward and reverse primers with all other reagents as described above. PCR reactions with the $36 \mathrm{~B} 4$ primers were thermal cycled for $95^{\circ} \mathrm{C}$ for $2 \mathrm{~min}$, followed by 35 cycles of $94^{\circ} \mathrm{C}$ for $30 \mathrm{~s}, 52^{\circ} \mathrm{C}$ for $30 \mathrm{~s}$, and $72^{\circ} \mathrm{C}$ for $30 \mathrm{~s}$ with a final extension for 5 min at $72^{\circ} \mathrm{C}$ and signal acquisition at the end of the $72^{\circ} \mathrm{C}$ step and melt curves generated by increasing temperatures from 72 to $95^{\circ} \mathrm{C}$, in $0.5^{\circ} \mathrm{C}$ steps, with a $30 \mathrm{~s}$ dwell period per step at the end of the thermal cycling.

All samples were PCR amplified in triplicate along with three no template controls per primer pair. Triplicates were averaged and any sample with a quantification cycle (Cq) standard deviation of greater than 0.5 between triplicates or with Cq values outside of the standard curve were repeated. Concentrations (ng) of telomere repeats and UCEs were determined using an external standard curve approach and Roche LightCycler software (release 4.0) for Absolute Quantification auto-analysis with 
the second derivative maximum method (proprietary). The efficiencies of each primer pair were determined from the slope of the standard curves using Roche LightCycler software where

$E=10^{[-1 / \text { slope }]}$. Optimal efficiency $(100 \%)$ is defined as a slope of 3.32. The correlation coefficients $\left(R^{2}\right)$ were determined from the replicates of the dilution series.

\section{Data analysis}

Primer pairs were assessed for their effect on stability values computationally (geNorm) as previously described (29). The same genomic DNA samples from multiple individuals were amplified with 3-5 UCE primer pairs, and the Cq values were used to sequentially eliminate the least stable primer pair. The two most stable UCE primer pairs were then used to generate DNA concentrations (ng) from a standard curve and these concentrations were graphed on a scatter plot with Pearson correlations and trend lines reported. To calculate relative telomere lengths in adult and nestling kestrels, the telomere concentration $(T)$ was divided by the average concentration of two UCE genes (UCE $E_{\text {ave }}$ ) to yield T/UCE ave.

\section{RESULTS}

\section{Assay development}

We set out to develop primer pairs to several UCEs to enable cross validation. We chose several UCEs from UCbase 2.0 and designed multiple primer pairs for each using a primer design tool (OligoArchitect, Sigma-Aldrich). During design, we limited the product length to $250 \mathrm{bps}$ and designed primers to have a $\mathrm{Tm}$ of $60^{\circ} \mathrm{C}$ to match the $\mathrm{Tm}$ of established telomere-specific primers (30). We ranked the primer pairs computationally (Beacon Designer) and experimentally tested the top four pairs for each of the UCEs by amplifying mouse DNA in a qPCR reaction. Using melt-curve analysis, we identified primer pairs for five of the UCEs that produced a single-peaked melt curve (Figure 1B). We determined the amplification efficiency for each of these five UCE primer pairs by amplifying seven serial dilutions of the mouse DNA in triplicate (Figure 1C). A primer pair for all five UCEs was found that have QPCR efficiencies within the best-practice range of $90-110 \%$ and correlations $\left(R^{2}\right)$ of the Cq values for replicates greater than or equal to 0.980 (31) (Figure 1C). The sequences of the chosen UCE primer pairs is reported in Table 1. 


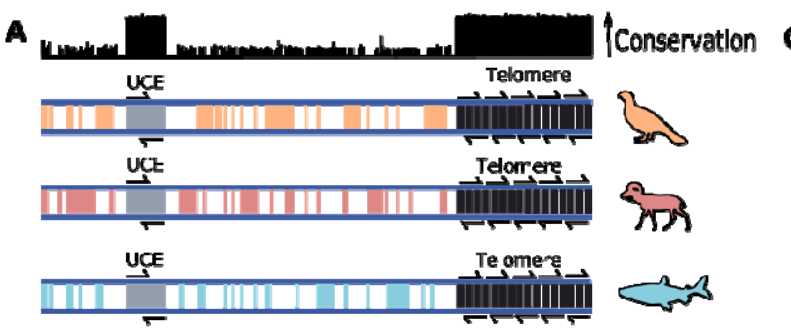

\begin{tabular}{|c|c|c|}
\hline UCE & Efficiency & $R^{2}$ \\
\hline UCE.359 & $97 \%$ & 0.99 \\
\hline UCE. 28 & $95 \%$ & 0.99 \\
\hline UCE.64 & $101 \%$ & 0.99 \\
\hline UCE. 239 & $101 \%$ & 0.99 \\
\hline UCE.176 & $102 \%$ & 0.99 \\
\hline
\end{tabular}

$\mathbf{B}$
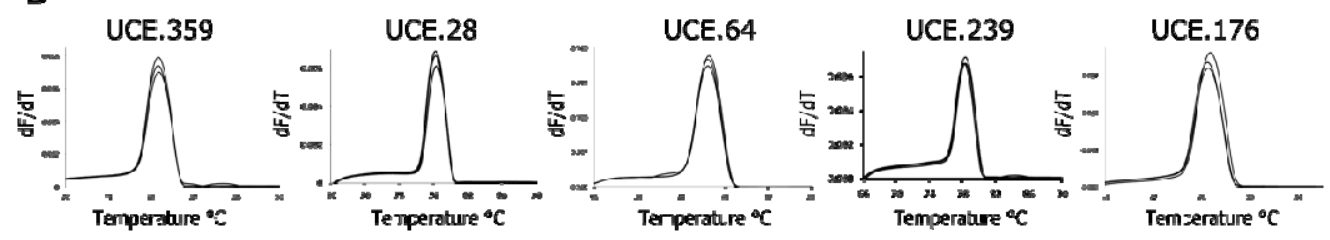

Figure 1. UCE primer performance in qPCR. (A) Conceptual diagram of the universal vertebrate telomere assay design. Black bars represent the level of conservation of genomic regions determined by the alignment of multiple genomes such as a bird, a mammal and a fish. Telomeric repeats are conserved in all chordates and are amplified by primers specific to this sequence. The same reference primers can be used in all species because they amplify ultra-conserved elements (UCE) that have high conservation between distant taxa. (B) Melt curves of the amplification product of the qPCR of genomic mouse DNA using the best primer pair for each UCE. (C) Efficiencies and $\mathrm{R}^{2}$ values of the best primer pair identified for five different UCEs, named by their access number in the UCE database.

Table 1 UCE and Telomere Primer Sequences

\begin{tabular}{ll}
\hline Name & Sequence \\
\hline tel 1b & CGGTTTGTTTGGGTTTGGGTTTGGGTTTGGGTTTGGGTT \\
\hline tel 2b & CAGCCGAAAGGCCCTTGGCAGGAGGGCTGCTGGTGGTCTACCCTT \\
\hline UCE.359-F & ATCTGAGACTTGTGACAT \\
\hline UCE.359-R & GTGTTAATTGGTAATGACTATT \\
\hline UCE.28-F & AAATACCACCCAACAGTTT \\
\hline UCE.28-R & AAGCCCTATACAGATGGAT \\
\hline UCE.64-F & GAGTCTCCAATATCATCAGAAGC \\
\hline UCE.64-R & ACACATGCCACGATCAATG \\
\hline UCE.239-F & TCAGATGTTCAGCCTATT \\
\hline UCE.239-R & AATACCATGTTAATATCCTCAA \\
\hline UCE.176-F & TTTCTACAGTTCTGATTTAGTTGA \\
\hline UCE.176-R & TGTTCCCTGTCGCATTAG \\
\hline
\end{tabular}




\section{Validation across the vertebrate tree of life}

We next tested our primers across the vertebrate tree of life (Figure 2A). We collected various tissue types from 19 species for DNA extraction. To test our primers as broadly as possible, we also included a sea squirt, which is amendable to telomere length measurements because basal chordates have the same telomeric repeat sequences as vertebrates. We performed qPCR on each sample in triplicate with all five primer pairs and evaluated the melt-curves. We found that all five primer pairs amplified DNA from every species with a single melt peak. All of the UCE primer pairs also have efficiencies in the best practice range of $90-110 \%$ when used to generate a standard curve through serial dilution (32). These results indicate that all five primer pairs are suitable primers for QPCR in all vertebrates that we tested.

An additional requirement for reference genes used for normalizing telomere length is that they must not vary in copy number among individuals in the population. For example, a duplication of a reference gene in one individual would appear as a halving of their telomere lengths relative to a non-duplicated individual. The amplification of closely related homolog variants could also introduce normalization differences that would have the same effect as copy number variation. The challenge of identifying reference primers with minimal variation between individuals for telomere length measurements is similar to choosing primer pairs for normalizing real-time PCR data for gene expression. We therefore evaluated our primer pairs using an algorithm, geNorm, designed for this purpose (29). The geNorm algorithm evaluates the cumulative variation among multiple primer pairs and iteratively eliminates primer pairs that contribute the most variation until final primer pairs are chosen. Two or more primer pairs are preferred over a single primer pair to reduce random experimental variation (33). Using the NormqPCR package in $R$ which utilizes the geNorm algorithm, we identified the best combination of UCE primer pairs from DNA extracted from 20 mice (Mus musculus), 17 rock ptarmigan (Lagopus muta), and 20 northern goshawks (Accipiter gentilis), representing both sexes and a range of ages. Samples were amplified by qPCR with each of the five reference primer sets using the same DNA concentration $(8 \mathrm{ng})$ for each reaction. We found that the use of two primer pairs for normalization leads to very low stability values in each of the species tested (Figure 2B). Next, we extracted DNA from another mammal (bighorn sheep, Ovis canadensis) and tested the top three primer pairs which had been identified in mouse. Using NormqPCR we identified the two primer pairs with the lowest stability values (Figure 2B). The genomic DNA concentration quantified by these two primer pairs showed high correlation (Pearson), suggesting that neither pair exhibit copy number polymorphisms within the individuals tested (Figure 2C and Supplementary Figure S1).

We next set out to determine if the UCE-based assay yields expected relative telomere length measurements in a species where telomere lengths have not been previously measured. We extracted DNA from the blood of 15 adult and 29 nestling American kestrels. We amplified each DNA sample separately with telomere specific primers, UCE.28 and UCE.239 which were chosen because of low stability values and high correlations. Relative telomere length was calculated as the ratio of 
the telomere amplification product to the average of the UCE amplification products. The results showed that adult American kestrels had a significantly shorter telomere length than nestlings, which was expected since telomeres tend to shorten with aging (Figure 2D). We note that, prior to developing the UCE primers, we had attempted to utilize published GAPDH primers to normalize telomere lengths in the American kestrel. We found that both the published (34) and extensively redesigned and optimized GAPDH primers exhibited abnormal PCR variations between individuals, making this commonly used reference gene unsuitable in this organism (Supplementary Figure S2). These results suggest that our primers may even be an improvement for telomere assays in avian species. 


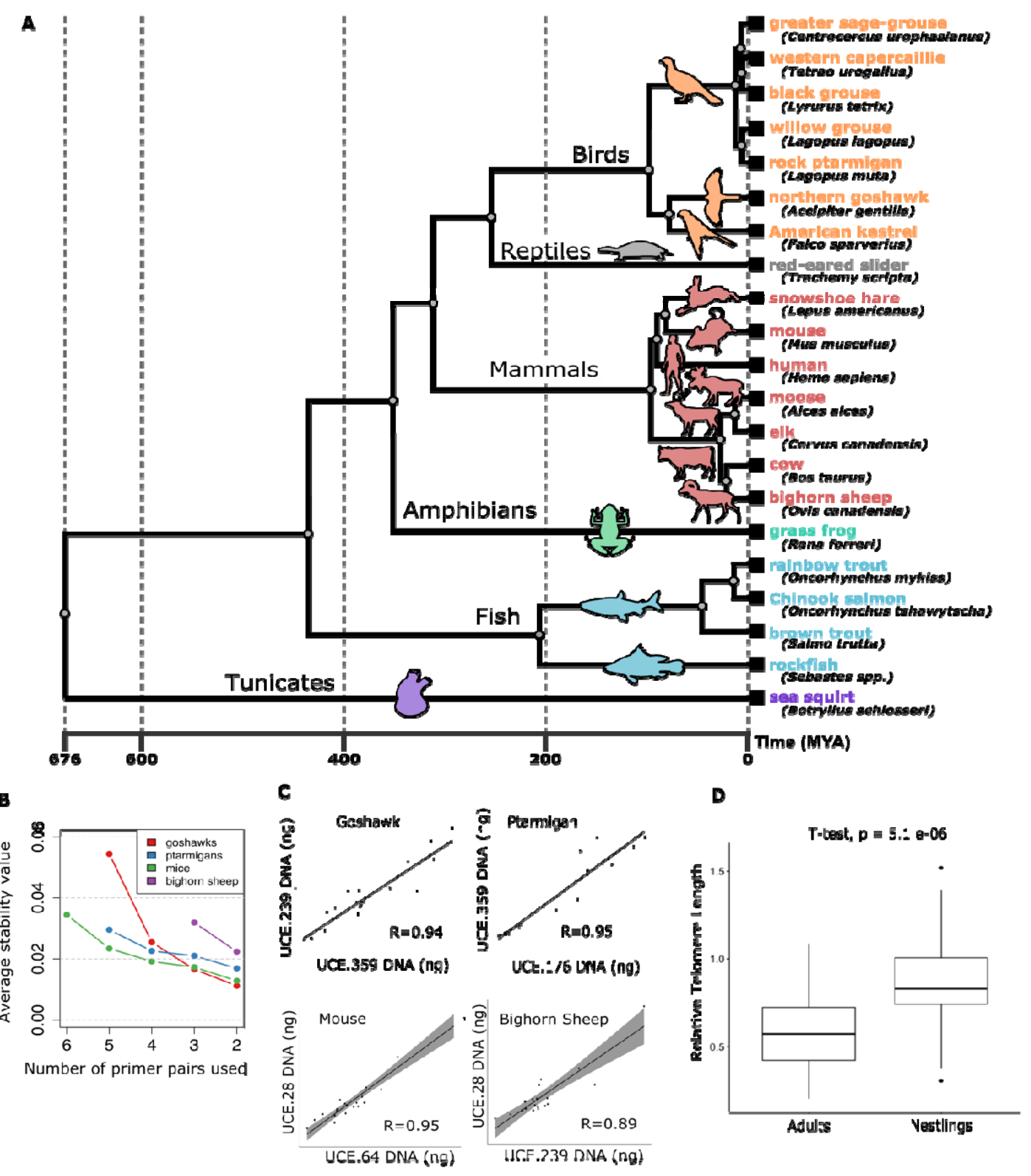

Figure 2. Validation of UCE reference primers across the vertebrate tree of life. (A) Phylogenetic tree of organisms in which reference primers were validated. (B) Average stability values generated in geNorm from the utilization of six to two reference gene primers. (C) Pearson correlation between the genomic DNA concentrations (ng) of the two UCE primer pairs that resulted in the lowest average stability values (geNorm) in each of four organisms. (D) Relative telomere length for adult and nestling American kestrels using UCE primers. 


\section{UCE primers are an improvement over previously reported telomere assay reference primers for mice}

We next quantified 20 mouse samples with a reference primer pair previously reported in the literature that was designed for qPCR-based telomere length measurement in mice (35). The previously reported primers target the acidic ribosomal phosphoprotein PO (36B4) gene and have been used in multiple publications (35). We used the published thermal cycling profile of the 36B4 primers to determine the PCR efficiency and evaluate the melt peak. The 36B4 primers had a good efficiency (102\%) but showed a broad, multi-peaked melt-curve indicating PCR artifacts or non-specific amplification occurred (Supplementary Figure S3). When we included the 36B4 primer pair in the NormqPCR stability analysis for mouse, this primer pair was the second eliminated by the geNorm algorithm, indicating that our top UCE primer pairs are an improvement with respect to genomic reference stability values in mice. These results further suggest that our UCE reference primers may be useful even in organisms with established reference primers.

\section{DISCUSSION}

Real-time qPCR-based telomere length assays provide a reliable and high-throughput method for studying telomeres. The primers reported here make this assay possible in any vertebrate species. For investigators that wish to study telomeres in a new organism, we recommend first testing all five UCE primer pairs on at least ten individuals and performing a stability analysis to find the best two primer pairs (29). Compared to a single reference primer pair, using the average quantity from two reference primer pairs for relative telomere length measurement has the advantage of averaging out other sources of qPCR noise (36). While three or more reference primers could be used if warranted, this would require more DNA and reagents. We were able to identify primer pairs with low stability values in bighorn sheep from comparing the best three primer pairs identified from mouse DNA samples (UCE.28, UCE.64 and UCE.239). This suggests that these three primers could be a good starting point for any mammalian or ungulate species. However, the slightly lower correlation between the concentrations measured for the bighorn sheep samples, compared to the other organisms, suggests that a better primer pair might be found if all five primers were tested (Figure $2 \mathrm{C}$ ). The bighorn sheep samples also had relatively low DNA concentration, which may also have contributed to the lower correlation value. We also recommend that in telomere assays in which all samples do not fit on one plate that the raw $T / U C E_{\text {ave }}$ ratio would be divided by the average $T / U C E_{\text {ave }}$ ratio of internal calibrator samples. In addition to primer choice, it is also important to establish appropriate tissue collection protocols for each new species because telomere length dynamics can vary between different types of cells and tissue (37). The biological relevance of telomere lengths must be determined in each organism which will require additional information such as diseases state and life history.

The ability to determine relative telomere length in any vertebrate species creates new opportunities in basic and applied research. For example, telomere length measurements can be 
used for monitoring the health and aging of organisms. Telomere lengths have even been considered a proxy for fitness because telomere lengths have been shown to predict lifespan and reproductive success in some species $(5,7)$. For organisms of conservation concern, telomere lengths have been shown to be an early indicator of extinction risk (9). Telomere lengths in indicator species in different environments may allow identification of important ecosystems disturbances, including those caused by humans. The ability to study multiple species simultaneously opens up questions about how telomere lengths change during long-term predator pray cycles or other ecological interactions. The ability to study telomere lengths in diverse taxa will allow for a better understanding of aging across the tree of life and from an evolutionary perspective (38).

\section{AVAILABILITY}

geNorm is open source and available in the R software package NormqPCR (https://github.com/jimrperkins/NormqPCR).

\section{ACKNOWLEDGEMENT}

We would like to thank Boise State University's Raptor Research Center, the Fulbright program, the Department of Biological Sciences, College of Arts and Sciences, and Division of Research, American kestrel nest box adopters, and landowners who have nest-boxes on their property as well as the Minidoka Ranger District of Sawtooth National Forest and Idaho Fish and Game. We also would like to acknowledge Jack Connelly (for sage-grouse), Örjan Johansson and Gier Rune Rauset (for capercaillie and black grouse), Rolf Brittas and Tomas Willebrand (for willow grouse), Knut Keiland (for snowshoe hares), Henrik Andrén and the Grimsö Wildlife Research Station (for moose).

\section{FUNDING}

The authors acknowledge support from the National Science Foundation [1826801 to J.B., J.F. and E.H.] National Science foundation [1807809 to E.H. and S.B.], as well the Semiconductor Research Corporation [2018-SB-2842 to E.H. and S.B.] and by the National Aeronautics and Space Administration [80NSSC17K0738 to E.H.]. Additional funding was provided through two National Science Foundation Research Experience for Undergraduates awards through the Division for Biological Infrastructure [1263167 to K.C. and J.H.] and the Division of Environmental Biology [1145552 to K.C. and J.H.]. Funding for open access charge: National Science Foundation [1826801].

\section{CONFLICT OF INTEREST}

None declared. 


\section{REFERENCES}

1. Gomes,N.M.V., Shay,J.W. and Wright,W.E. (2010) Telomere Biology in Metazoa. FEBS Lett., 584, 3741-3751.

2. O’Sullivan,R.J. and Karlseder,J. (2010) Telomeres: protecting chromosomes against genome instability. Nat. Rev. Mol. Cell Biol., 11, 171-181.

3. McLennan,D., Armstrong,J.D., Stewart,D.C., Mckelvey,S., Boner,W., Monaghan,P. and Metcalfe,N.B. (2016) Interactions between parental traits, environmental harshness and growth rate in determining telomere length in wild juvenile salmon. Mol. Ecol., 25, 5425-5438.

4. Wilbourn,R.V., Moatt,J.P., Froy,H., Walling,C.A., Nussey,D.H. and Boonekamp,J.J. (2018) The relationship between telomere length and mortality risk in non-model vertebrate systems: a meta-analysis. Philos. Trans. R. Soc. B Biol. Sci., 373.

5. Bauch,C., Becker,P.H. and Verhulst,S. (2013) Telomere length reflects phenotypic quality and costs of reproduction in a long-lived seabird. Proc R Soc B, 280, 20122540.

6. Saino,N., Romano,M., Ambrosini,R., Rubolini,D., Boncoraglio,G., Caprioli,M. and Romano,A. (2012) Longevity and lifetime reproductive success of barn swallow offspring are predicted by their hatching date and phenotypic quality. J. Anim. Ecol., 81, 1004-1012.

7. Eastwood,J.R., Hall,M.L., Teunissen,N., Kingma,S.A., Hidalgo Aranzamendi,N., Fan,M., Roast,M., Verhulst,S. and Peters,A. (2019) Early-life telomere length predicts lifespan and lifetime reproductive success in a wild bird. Mol. Ecol., 28, 1127-1137.

8. Heidinger,B.J., Blount,J.D., Boner,W., Griffiths,K., Metcalfe,N.B. and Monaghan,P. (2012) Telomere length in early life predicts lifespan. Proc. Natl. Acad. Sci., 109, 1743-1748.

9. Dupoué,A., Rutschmann,A., Le Galliard,J.F., Clobert,J., Angelier,F., Marciau,C., Ruault,S., Miles,D. and Meylan,S. (2017) Shorter telomeres precede population extinction in wild lizards. Sci. Rep., 7, 16976.

10. Bauch,C., Boonekamp,J.J., Korsten,P., Mulder,E. and Verhulst,S. (2019) Epigenetic inheritance of telomere length in wild birds. PLOS Genet., 15, e1007827.

11. Tricola,G.M., Simons,M.J.P., Atema,E., Boughton,R.K., Brown,J.L., Dearborn,D.C., Divoky,G., Eimes,J.A., Huntington,C.E., Kitaysky,A.S., et al. (2018) The rate of telomere loss is related to maximum lifespan in birds. Philos. Trans. R. Soc. B Biol. Sci., 373.

12. Barnes,R.P., Fouquerel,E. and Opresko,P.L. (2019) The impact of oxidative DNA damage and stress on telomere homeostasis. Mech. Ageing Dev., 177, 37-45.

13. Martens,D.S. and Nawrot,T.S. (2016) Air Pollution Stress and the Aging Phenotype: The Telomere Connection. Curr. Environ. Health Rep., 3, 258-269. 
14. McLennan,D., Armstrong,J.D., Stewart,D.C., Mckelvey,S., Boner,W., Monaghan,P. and Metcalfe,N.B. (2016) Interactions between parental traits, environmental harshness and growth rate in determining telomere length in wild juvenile salmon. Mol. Ecol., 25, 5425-5438.

15. Meillère,A., Brischoux,F., Ribout,C. and Angelier,F. (2015) Traffic noise exposure affects telomere length in nestling house sparrows. Biol. Lett., 11, 20150559.

16. Bauch,C., Becker,P.H. and Verhulst,S. (2014) Within the genome, long telomeres are more informative than short telomeres with respect to fitness components in a longlived seabird. Mol. Ecol., 23, 300-310.

17. Beaulieu,M., Reichert,S., Le Maho,Y., Ancel,A. and Criscuolo,F. (2011) Oxidative status and telomere length in a long-lived bird facing a costly reproductive event. Funct. Ecol., 25, 577-585.

18. Quirici,V., Guerrero,C.J., Krause,J.S., Wingfield,J.C. and Vásquez,R. a. (2016) The relationship of telomere length to baseline corticosterone levels in nestlings of an altricial passerine bird in natural populations. Front. Zool., 10.1186/s12983-0160133-5.

19. Martin-ruiz,C.M., Baird,D., Roger,L., Boukamp,P., Krunic,D., Cawthon,R., Dokter,M.M., Harst,P.V.D., Bekaert,S., Meyer,T.D., et al. (2015) Reproducibility of telomere length assessment $\square$ : an international collaborative study. 10.1093/ije/dyu191.

20. Lin,J., Smith,D.L., Esteves,K. and Drury,S. (2019) Psychoneuroendocrinology Telomere length measurement by qPCR - Summary of critical factors and recommendations for assay design. Psychoneuroendocrinology, 99, 271-278.

21. Cawthon,R.M. (2002) Telomere measurement by quantitative PCR. Nucleic Acids Res., 30, e47.

22. Meyne,J., Ratliff,R.L. and Moyzis,R.K. (1989) Conservation of the human telomere sequence (TTAGGG)n among vertebrates. Proc. Natl. Acad. Sci., 86, 7049-7053.

23. Smith,S., Turbill,C. and Penn,D.J. (2011) Chasing telomeres, not red herrings, in evolutionary ecology. Heredity, 107, 372-373.

24. Horn,T., Robertson,B.C. and Gemmell,N.J. (2010) The use of telomere length in ecology and evolutionary biology. Heredity, 105, 497-506.

25. Bejerano,G. (2004) Ultraconserved Elements in the Human Genome. Science, 304, 13211325.

26. McCormack,J.E., Faircloth,B.C., Crawford,N.G., Gowaty,P.A., Brumfield,R.T. and Glenn,T.C. (2012) Ultraconserved elements are novel phylogenomic markers that resolve placental mammal phylogeny when combined with species-tree analysis. Genome Res., 22, 746-754.

27. Asghar,M., Hasselquist,D. and Bensch,S. (2011) Are chronic avian haemosporidian infections costly in wild birds? J. Avian Biol., 42, 530-537. 
28. Bustin,S.A., Benes, V., Garson,J.A., Hellemans,J., Huggett,J., Kubista,M., Mueller,R., Nolan,T., Pfaffl,M.W., Shipley,G.L., et al. (2009) The MIQE guidelines: minimum information for publication of quantitative real-time PCR experiments. Clin. Chem., 55, 611-622.

29. Vandesompele,J., De Preter,K., Pattyn,F., Poppe,B., Van Roy,N., De Paepe,A. and Speleman,F. (2002) Accurate normalization of real-time quantitative RT-PCR data by geometric averaging of multiple internal control genes. Genome Biol., 3, research0034.1.

30. Roos,G., Kröber,A., Grabowski,P., Kienle,D., Bühler,A., Döhner,H., Rosenquist,R. and Stilgenbauer,S. (2008) Short telomeres are associated with genetic complexity, highrisk genomic aberrations, and short survival in chronic lymphocytic leukemia. Blood, 111, 2246-2252.

31. Kuang,J., Yan,X., Genders,A.J., Granata,C. and Bishop,D.J. (2018) An overview of technical considerations when using quantitative real-time PCR analysis of gene expression in human exercise research. PLOS ONE, 13, e0196438.

32. ROGERS-BROADWAY,K.-R. and KARTERIS,E. (2015) Amplification efficiency and thermal stability of qPCR instrumentation: Current landscape and future perspectives. Exp. Ther. Med., 10, 1261-1264.

33. D'haene,B., Vandesompele,J. and Hellemans,J. (2010) Accurate and objective copy number profiling using real-time quantitative PCR. Methods, 50, 262-270.

34. Criscuolo,F., Bize,P., Nasir,L., Metcalfe,N.B., Foote,C.G., Griffiths,K., Gault,E.A. and Monaghan,P. (2009) Real-time quantitative PCR assay for measurement of avian telomeres. J. Avian Biol., 40, 342-347.

35. Callicott,R.J. and Womack,J.E. (2006) Real-time PCR assay for measurement of mouse telomeres. Comp. Med., 56, 17-22.

36. Kozera,B. and Rapacz,M. (2013) Reference genes in real-time PCR. J. Appl. Genet., 54, 391-406.

37. Lin,J., Smith,D.L., Esteves,K. and Drury,S. (2019) Telomere length measurement by qPCR - Summary of critical factors and recommendations for assay design. Psychoneuroendocrinology, 99, 271-278.

38. Gomes,N.M.V., Ryder,O.A., Houck,M.L., Charter,S.J., Walker,W., Forsyth,N.R., Austad,S.N., Venditt,C., Pagel,M., Shay,J.W., et al. (2011) Comparative biology of mammalian telomeres: hypotheses on ancestral states and the roles of telomeres in longevity determination. Aging Cell, 10, 761-768. 